Revue des sciences de l'eau

\title{
Estimation des ressources en eau superficielle aux pas de temps mensuel et annuel, application à un pays semi-aride Monthly water resources assessment, application to a semi-arid country
}

\author{
M. Kabouya et C. Michel
}

Volume 4, numéro 4, 1991

URI : https://id.erudit.org/iderudit/705116ar

DOI : https://doi.org/10.7202/705116ar

Aller au sommaire du numéro

Éditeur(s)

Université du Québec - INRS-Eau, Terre et Environnement (INRS-ETE)

ISSN

0992-7158 (imprimé)

1718-8598 (numérique)

Découvrir la revue

Citer cet article

Kabouya, M. \& Michel, C. (1991). Estimation des ressources en eau superficielle aux pas de temps mensuel et annuel, application à un pays semi-aride. Revue des sciences de l'eau / Journal of Water Science, 4(4), 569-587.

https://doi.org/10.7202/705116ar
Résumé de l'article

On connait l'extrême variabilité des ressources en eau superficielle et les problèmes que cela pose en aménagement du territoire. En conséquence, générer de longues séries de débits annuels (ou mensuels) est une nécessité pour l'étude de la disponibilité des ressources en eau (BURGES et LETTENMAIER, 1977).

Cette génération de longues séries peut se faire à partir de la connaissance des pluies si l'on dispose d'un modèle fiable de transformation pluie-débit. Un premier modèle linéaire visant à l'estimation du seul débit annuel, déjà utile pour un premier dégrossissage de la ressource, est bâti à partir de la pluie annuelle et de sa répartition entre les mois. Il constitue un outil de base que l'on a jugé néanmoins un peu trop rudimentaire. La recherche d'un modèle conceptuel simple nous a semblé une voie plus prometteuse. Exploitant une recherche déjà entreprise sur ce type de modèle au pas de temps journalier, on a construit un modèle mensuel à trois paramètres dont l'élaboration a été justifiée point par point par une meilleure efficacité des résultats fournis sur une soixantaine de bassins versants appartenant à la partie septentrionale de l'Algérie. Nous avons analysé les paramètres du modèle et obtenu une estimation approchée de la matrice des variances-covariances de ces paramètres afin de disposer d'un outil similaire à celui existant pour le modèle linéaire général.
Tous droits réservés (C) Revue des sciences de l'eau, 1991
Ce document est protégé par la loi sur le droit d'auteur. L'utilisation des services d'Érudit (y compris la reproduction) est assujettie à sa politique d'utilisation que vous pouvez consulter en ligne.

https://apropos.erudit.org/fr/usagers/politique-dutilisation/ 


\title{
Estimation des ressources en eau superficielle aux pas de temps mensuel et annuel, application à un pays semi-aride
}

\author{
Monthly water resources assessment, \\ application to a semi-arid country
}

M. KABOUYA 1 et C. MICHEL 2

Reçu le 16 juillet 1990, accepté pour publication le 9 juillet $1991^{*}$.

RÉSUMÉ

On connaît l'extrême variablití des ressources en eau superficielle et les problèmes que cela pose en aménagement du territoire. En conséquence, générer de longues séries de débits annuels (ou mensuels) est une nécessité pour l'étude de la disponibillté des ressources en eau (BURGES et LETTENMAIER, 1977).

Cette génération de longues séries peut se faire à partir de la connaissance des pluies si l'on dispose d'un modẻie fiable de transformation pluie-débit. Un premier modèle linéaire visant à l'estimation du seul déblt annuel, déjà utile pour un premier dégrossissage de la ressource, est bati à partir de la pluie annuelle et de sa répartition entre les mois. II constitue un outil de base que l'on a jugé néanmoins un peu trop rudimentaire. La recherche d'un modèle conceptuel simple nous a semblé une voie plus prometteuse. Exploitant une recherche déjà entreprise sur ce type de modèle au pas de temps journalier, on a construit un modèle mensuel à trois paramètres dont l'élaboration a été justifiée point par point par une meilleure efficacité des résultats fournis sur une soixantalne de bassins versants appartenant a la partie septentrionale de I'Algérle. Nous avons analysé les paramètres du modèle el obtenu une estimation approchée de la matrice des variances-covariances de ces paramètres afin de disposer d'un outil similaire à celul existant pour le modèle linéaire général.

Mots clés : ressources en eau, modèle conceptuel, pas de temps mensuel et annuel, sensibilité des paramètres.

1. Stagiaire en thèse au CEMAGREF, Division Hydrologie, BP 121, 92185 Antony, France.

2. Ingénieur à la Division Hydrologie du CEMAGREF.

* Les commentaires seront reçus jusqu'au 15 septembre 1992. 


\section{SUMMARY}

Availability of long series of annual or monthly streamilows appears to be a necessity in water resources studies. In regions where such data do not exist we may resort to rainfall data to obtain the required tong series of streamflows. In this case we need a reliable rainfall-runoff model. Such a model is often called a water balance model although this designation ignores the dynamics involved in the model. A first attempt was to establish a linear model connecting an annual streamflow to the concurrent rainfall and its allocation within the year.

This model was calibrated on 63 catchments throughout nothern Algeria, an area of about $200,000 \mathrm{~km}^{2}$ where mean annual streamflows range from 5 to 350 $\mathrm{mm}$.

The standard error of the model, expressed as a percentage of the mean annual streamflow was about $41 \%$. This relatively poor result led us to explore the capabilities of lumped conceptual models. Hydrologic models may be of the following three types:

- the linear black box model,

- the lumped conceptual model,

- the physically based model.

The linear black box model may benefit from huge mathematical developments but is very poor from a hydrologic point of view. The physically based model is a permanent search to comply more and more to the whole catchment and alt processes which are supposed to take place in it. It is tantamount to sheer utopia since the required fine description of the catchment will be definitely out of reach for economical reasons. The only workable solution lies in the design of a lumped conceptual model. But we have to bear in mind that such models cannot rely on a comprehensive physical basis and thus, all specifications have to be justified by the outputs of the model.

We benefited from a recent research upon a daily conceptual model that served as a starting sketch for the monthly model. After having tested numerous alternatives we worked out a simple model with only three parameters to be calibrated. The standard error of this model was about $27 \%$ of the mean annual flow. According to recent analyses about the sensitivity of model parameters, we calcutated the matrix of covariances of the parameters.

This was necessary because, due to the lack of a long series of data, we could not achieve an actual validation of the model against a streamflow sequence not used in the calibration process. However, the extreme parcimony of the model may alleviate the need of such a validation and the model compared favourably with well-known water balance models. As a conclusion, we may hope that the tool we have devised will be of valuable help in water resources management.

Key-words: water resources, monthly lumped models, parameter uncertainty. 


\section{NOTATIONS}

$A, B, C, D$ : paramètres d'un modèle conceptuel ;

$a, b, c$ : logarithmes des paramètres $A, B, C$;

$a_{0}, a_{1}, a_{2}$ : paramètres d'un modèle linéaire;

$\bar{E}$ : évapotranspiration potentielle mensuelle moyenne $(\mathrm{mm})$;

e : erreur d'un modèle ( $\mathrm{mm}$ ) :

g, $h$ : nombres fonctions de $s$, taux de remplissage d'un réservoir sol ;

$P$ : pluie mensuelle ;

$P^{\prime}$ : pluie mensuelle nette $(\mathrm{mm})$;

$P_{a}$ : pluie annuelle $(\mathrm{mm})$;

$P_{x}$ : pluie mensuelle maximale d'une année $(\mathrm{mm})$;

$Q$ : écoulement mensuel $(\mathrm{mm})$;

$Q_{0}, Q_{1}, Q_{2}, Q_{3}$ : écoulements mensuels calculés (mm);

$Q_{\mathrm{a}}$ : écoulement annuel (mm);

$\bar{Q}_{\mathrm{a}}$ : écoulement moyen interannuel $(\mathrm{mm})$;

$R$ : stock en eau d'un réservoir de a routage $\cdots(\mathrm{mm})$;

$S$ : stock en eau d'un réservoir * sol * $(\mathrm{mm})$ :

$s$ : taux de remplissage d'un réservoir sol $(s=S / A)$;

$\alpha, B, \gamma:$ paramètres d'un modèle linéaire ;

$\varepsilon$ : nombre ;

$\sigma:$ écart-type de l'erreur d'un modèle.

\section{1 - INTRODUCTION}

On se propose dans cet article de présenter une tentative d'estimation des ressources en eau de surface à un pas de temps très grand : pas de temps annuel et pas de temps mensuel.

La détermination des écoulements à ce niveau de globalisation temporelle est une des táches fondamentales des gestionnaires de la ressource en eau car beaucoup de problèmes relatifs à la confrontation des ressources et des besoins peuvent être dégrossis à cette échelle de temps. Citons également que certaines recherches sur l'étude des conséquences d'un éventuel réchauffement de la planète sur la disponibilité de la ressource en eau, ont recours à l'analyse mensuelle pour explorer le sujet (SCHAAKE et CHUNZHEN, 1989), (MCCABE et AYERS, 1989). Bien que des résultats au pas de temps annuel puissent être suffisants, leur obtention nécessite généralement le recours à des données mensuelles. Il est alors probablement plus efficace de 
bâtir d'emblée un modèle mensuel. C'est pourquoi l'essentiel du présent article porte sur l'établissement d'un modèle mensuel pluie-débit auquel on s'est efforcé de donner la simplicité la plus grande pour accroïtre la faisabilité utlérieure d'une estimation a priori des paramètres et donc d'une application du modèle à des bassins dépourvus de données. Cette recherche de la simplicité s'explique aussi par le fait que les données hydrologiques sont en général assez imprécises et ne permettent pas d'exploiter les potentialités de modèles un tant soit peu complexes.

\section{2 - MODĖLE LINÉAIRE ET PAS DE TEMPS ANNUEL}

Pour débuter notre étude, nous avons choisi de nous intéresser au pas de temps annuel en utilisant un modèle linéaire. Pour fixer ce modèle, nous supposons que l'information pluviomètrique est disponible au pas de temps mensuel. Nous avons cherché pour les raisons évoquées précédemment un modèle très simple c'est-à-dire utilisant deux ou trois variables explicatives. On se pose généralement la question de savoir si le modèle portera sur la lame écoulée ou sur une transformée logarithmique ou autre. Un désavantage parfois attribué au modèle linéaire portant sur le logarithme du débit est le biais qu'il introduit dans l'estimation des volumes écoulés moyens. En fait ce biais peut être corrigé si l'on tient compte de l'écart type de l'erreur du modèle soit dans une simulation soit dans le calcul des valeurs moyennes. Une discussion des différentes corrections possibles est donnée par GILROY et al. (1990). Les avantages du modèle logarithmique sont essentiellement, d'une part, le fait que l'on satisfait tout naturellement la contrainte de non-négativité des apports et d'autre part, que l'on considère une erreur relative et non pas absolue, ce qui permet de traiter avec une même précision relative faibles et forts apports.

En ce qui concerne le choix des variables explicatives, compte tenu des limites que nous nous sommes fixés, on ne peut guère introduire que le volume total et un indice de répartition. Après quelques essais nous avons retenu le modèle suivant :

$$
\text { In } Q_{a}=a_{0}+a_{1} \ln P_{a}+a_{2} \ln P_{x}+e
$$

dans lequel on $\mathbf{a}$ :

$Q_{a}=$ écoulement annuel $(\mathrm{mm})$,

$P_{a}=$ pluie annuelle $(\mathrm{mm})$,

$P_{x}=$ pluie mensuelle maximale de l'année $(\mathrm{mm})$,

$e=$ erreur du modèle d'écart-type $\sigma$,

In ()$=$ logarithme népérien,

$a_{0}, a_{1}, a_{2}=$ paramètres du modèle.

Pour pouvoir juger de la pertinence de ce modèle, il faut le confronter à des données observées, que nous présentons ci-après. 


\section{3 - Données utilisées}

L'étude des apports dont il est question dans ce document concerne l'Algérie septentrionale c'est-à-dire une région d'environ $200000 \mathrm{~km}^{2}$ dont la pluviométrie varie de 250 à $1800 \mathrm{~mm}$.

Des données mensuelles sont disponibles pour 63 bassins versants dont la taille varie de 70 a $2000 \mathrm{~km} 2$ et dont lécoulement moyen annuel s'étale de 5 à $350 \mathrm{~mm}$ environ. Leur situation dans l'Algérie septentrionale est illustrée par la figure 1 et leur liste apparaît dans le tableau 1. Les données sont relatives à des périodes d'observation assez courtes (5 à 20 ans environ, très irrégulièrement réparties entre 1947 à 1985) et assez variables d'un bassin à l'autre. Des lacunes existent dans les séries, seules les lacunes de courte durée (moins de 3 mois dans l'année) ont été comblées par corrélation spatiale. Cette situation est due aux discontinuités historiques dans les équipes techniques chargées de la gestion des données ainsi qu'aux difficultés spécifiques de mesure dans les régions aux écoulements faibles et intermittents. Il est certain que des données fragmentaires ne permettent pas de valider des modèles très exigeants et écartent généralement la possibilité de prévoir une validation en bonne et due forme d'un modèle par suite de la difficulté à séparer les observations en une période de calage et une période de contrôle de durées significatives. C'est en particulier pour cette raison que l'on est contraint de ne mettre en œuvre que des modèles très simples pour lesquels les risques de surcalage sont a priori exclus.

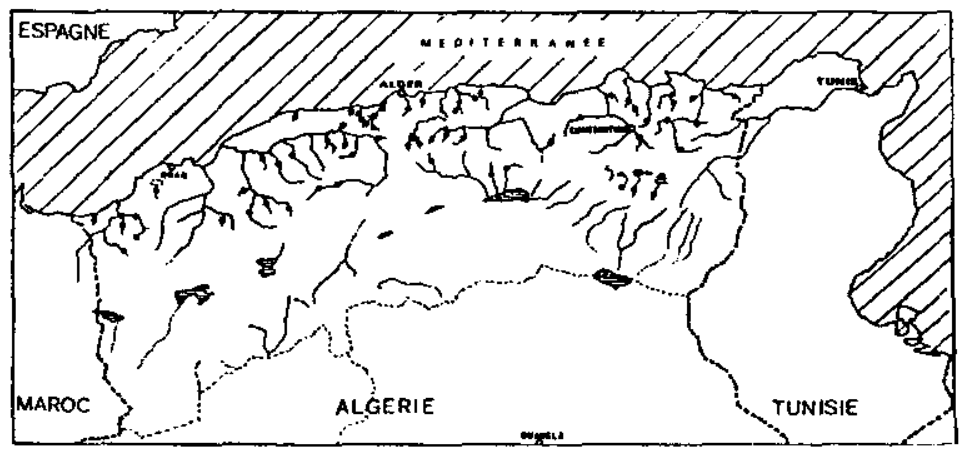

Figure 1 Plan de situation des 63 bassins versants (•) étudiés en Algérie septentrionale.

Vicinity map of the 63 catchments in Northern Algeria. 
Tableau 1 Liste des 63 stations hydrométriques utilisées

\begin{tabular}{|c|c|c|c|c|c|c|c|}
\hline $\begin{array}{l}\text { Cadre } \\
\text { toydrologique }\end{array}$ & $\begin{array}{l}\text { Nom } \\
\text { Station }\end{array}$ & $\begin{array}{c}\text { Mom } \\
\text { Cours treau }\end{array}$ & $\begin{array}{c}\text { Coordomnies: } \\
\mathbf{X}(\mathbf{k m})\end{array}$ & $\begin{array}{l}\text { Lambent } \\
Y(k n)\end{array}$ & $\begin{array}{c}\text { Surface } \\
\left(\mathbf{k m}^{2}\right)\end{array}$ & $\begin{array}{l}\text { Pluie } \\
\text { annuelle } \\
\text { moyenne } \\
\text { (mm) }\end{array}$ & $\begin{array}{l}\text { Ecou- } \\
\text { lement } \\
\text { annuel } \\
\text { moyen } \\
(\mathrm{mm})\end{array}$ \\
\hline $\begin{array}{l}04.01 .01 \\
04.04 .01 \\
16.04 .01 \\
16.06 .01 \\
16.07 .02 \\
16.07 .04 \\
16.07 .03 \\
11.1220 \\
01.09 .07 \\
01.10 .01 \\
01.15 .01 \\
01.16 .02 \\
01.17 .15 \\
01.18 .01 \\
01.19 .06 \\
01.23 .11 \\
01.26 .01 \\
01.27 .01 \\
01.29 .09 \\
01.30 .01 \\
01.33 .01 \\
01.34 .01 \\
02.02 .07 \\
02.03 .01 \\
02.03 .18 \\
02.10 .07 \\
02.10 .02 \\
0210.11 \\
02.11 .26 \\
02.12 .01 \\
02.13 .01 \\
02.06 .08 \\
0216.05 \\
02.18 .03 \\
02.19 .05 \\
02.19 .00 \\
09.01 .01 \\
09.03 .09 \\
09.03 .05 \\
15.01 .14 \\
15.01 .06 \\
15.02 .04 \\
15.04 .01 \\
15.06 .01 \\
10.01 .09 \\
10.05 .01 \\
10.07 .02 \\
03.07 .01 \\
03.07 .02 \\
03.08 .01 \\
03.11 .02 \\
03.11 .01 \\
03.16 .01 \\
14.02 .02 \\
14.03 .01 \\
14.05 .01 \\
14.06 .02 \\
07.07 .02 \\
07.04 .03 \\
07.03 .01 \\
05.09 .01 \\
05.08 .01 \\
05.01 .01\end{array}$ & 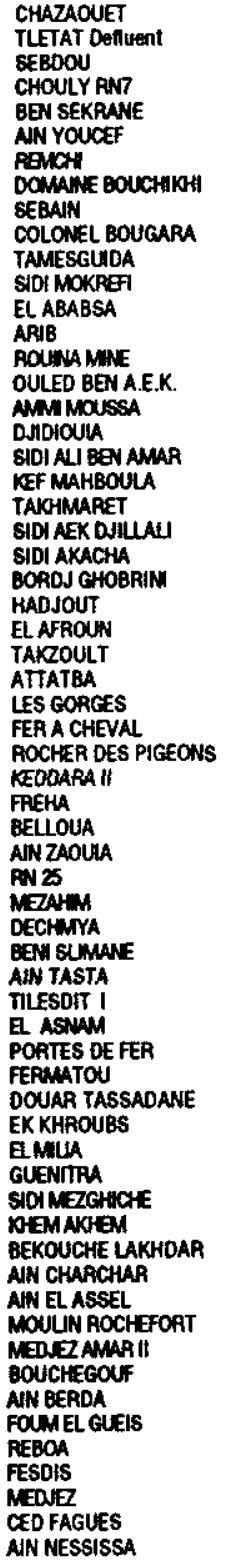 & 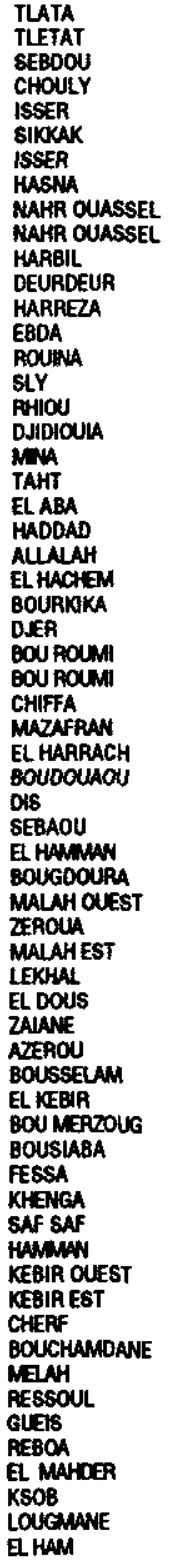 & 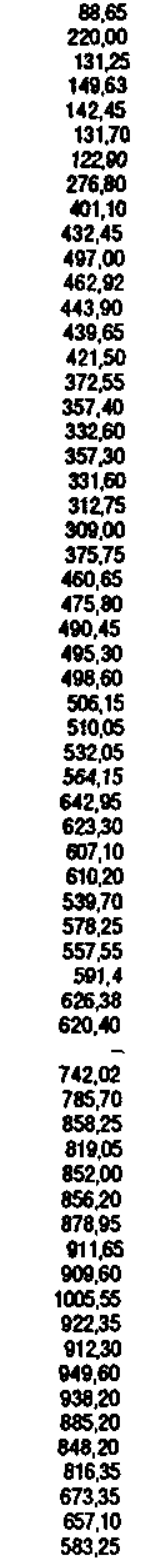 & $\begin{array}{r}205,30 \\
245,80 \\
158,57 \\
181,06 \\
204,65 \\
201,70 \\
208,85 \\
194,10 \\
239,75 \\
251,95 \\
323,90 \\
302,00 \\
318,50 \\
335,55 \\
324,50 \\
301,70 \\
286,35 \\
293,95 \\
225,65 \\
224,25 \\
202,75 \\
244,50 \\
354,30 \\
367,00 \\
358,80 \\
350,70 \\
347,90 \\
362,85 \\
342,30 \\
373,20 \\
354,75 \\
372,55 \\
384,25 \\
381,20 \\
363,50 \\
375,81 \\
322,65 \\
316,45 \\
322,25 \\
329,6 \\
340,58 \\
335,50 \\
329,0 \\
329,40 \\
359,40 \\
337,80 \\
391,00 \\
336,00 \\
382,40 \\
370,30 \\
387,45 \\
393,15 \\
400,00 \\
319,80 \\
358,75 \\
362,10 \\
387,00 \\
247,30 \\
250,20 \\
259,00 \\
289,65 \\
377,90 \\
290,35\end{array}$ & 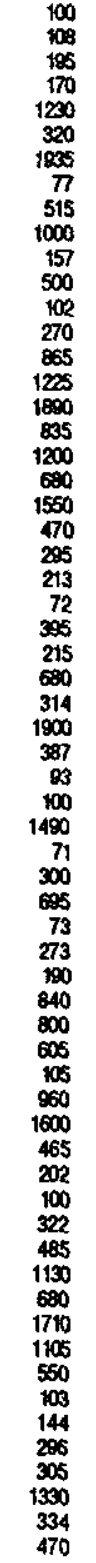 & $\begin{array}{l}448 \\
451 \\
498 \\
630 \\
577 \\
652 \\
593 \\
414 \\
406 \\
398 \\
732 \\
547 \\
486 \\
660 \\
524 \\
486 \\
544 \\
339 \\
449 \\
417 \\
281 \\
300 \\
631 \\
693 \\
683 \\
778 \\
712 \\
778 \\
600 \\
732 \\
706 \\
987 \\
1094 \\
1085 \\
719 \\
708 \\
331 \\
431 \\
339 \\
251 \\
367\end{array}$ & $\begin{array}{r}48 \\
21 \\
85 \\
90 \\
50 \\
87 \\
62 \\
20 \\
15 \\
17 \\
108 \\
84 \\
51 \\
313 \\
58 \\
115 \\
81 \\
25 \\
29 \\
21 \\
6 \\
13 \\
100 \\
222 \\
72 \\
105 \\
152 \\
138 \\
336 \\
120 \\
277 \\
356 \\
327 \\
335 \\
131 \\
223 \\
29 \\
103 \\
49 \\
75 \\
149 \\
31 \\
31 \\
119 \\
99 \\
6 \\
175 \\
172 \\
71 \\
118 \\
85 \\
113 \\
308 \\
17 \\
61 \\
219 \\
108 \\
74 \\
67 \\
23 \\
17 \\
24 \\
53\end{array}$ \\
\hline
\end{tabular}




\section{4 - PERFORMANCES DU MODĖLE LINÉAIRE}

La relation (1) a été testée sur les 63 bassins du cadre géographique de notre étude et l'on a constaté que le coefficient $a_{2}$ n'était pas toujours significativement différent de zéro, si l'on en juge par le rapport de Student de ce coefficient. Nous avons malgré tout recherché un groupement des deux variables $P_{a}$ et $P_{x}$ qui soit sensiblement plus efficace que $P a$ pris isolément. Cette démarche a abouti au modèle très simple à deux paramètres qui suit :

$$
\text { In } Q_{a}=a_{0}+a_{1}\left(\ln P_{a}+0,25 \ln P_{x}\right)+e
$$

Ce modèle donne cependant sur onze bassins un résultat décevant puisque le rapport de Student de la variable explicative y prend une valeur inférieure à 3.

Il est intéressant de convertir l'erreur standard du modèle logarithmique en erreur absolue exprimée en $\mathrm{mm}$ ou mieux encore en pourcentage du module (débit moyen interannuel $\bar{Q}_{\mathrm{a}}$ ). Si l'on procède ainsi on obtient une erreur standard variant trés largement entre 10 et $120 \%$ de $\bar{Q}_{a}$, avec pour valeur moyenne $41,4 \%$.

Nous reprendrons plus loin ce critère global pour l'ensemble des 63 bassins versants étudiés et nous le désignerons par les termes * critère annuel moyen .

Le modèle linéaire permet une détermination des écoulements annuels, certes utile, mais bien imprécise pour une exploitation opérationnelle efficace. En ce qui concerne le coefficient $a_{1}$ relatif à la variable explicative pluviométrique, on a constaté qu'il variait en gros de 0,5 à 4 avec une médiane égale à 2. Avec l'espoir d'obtenir des estimations plus précises pour les écoulements, nous avons essayé une modélisation moins radicale que la boîte noire linéaire.

\section{5 - MODÉLISATION CONCEPTUELLE AU PAS DE TEMPS MENSUEL}

La modélisation hydrologique oscille entre trois pôles qui sont :

- la boîte noire linéaire,

- la modélisation fondée sur la physique.

- la modélisation conceptuelle globale.

La modélisation linéaire bénéficie d'un précieux arsenal mathématique mais affiche une grande pauvreté dans la représentation des phénomènes hydrologiques du fait de la structure très sommaire des modèles. La modélisation d'inspiration physique consiste à décrire de la façon la plus fidèle possible, les processus qui se déroulent dans un bassin, en s'appuyant sur 
des mesures physiques concernant les sols et le substratum. La logique de cette approche physique est d'affiner indéfiniment le modèle jusqu'à retrouver le prototype. Jusqu'à présent elle a toujours échoué, non pas du fait que les outils manquaient pour décrire ces processus mais tout simplement parce que la description réelle du système où se produisent ces processus est hors de portée pour un coût raisonnable. Dans ces conditions, on se trouve contraint de progresser dans cette troisième voie que constitue la modélisation conceptuelle globale. Le qualificatif "conceptuel * rappelle que l'on recherche des schémas respectant une certaine logique du processus pluiedébit qui exige, entre autres, que les sorties en écoulement de surface soient intérieures aux entrées par les pluies. Le qualificatif « global " signifie que l'on considère le bassin versant comme un tout, sans analyser ses différentes parties, ce qu'on ne saurait faire de façon efficace autrement que par une approche physique.

Ces précisions étant apportées, on comprendra qu'un modèle conceptuel global ne peut être qu'empirique et qu'en conséquence, chaque spécification qu'il contient doit être validée (et ne peut être validée que) par une représentation des débits observés très sensiblement plus satisfaisante que si cette spécification en question était absente. Peut-être pourra-t-on objecter que cette démarche n'est que du a curve fitting " mais c'est effectivement la seule démarche possible pour les modèles conceptuels.

Au pas de temps mensuel, les modèles conceptuels globaux sont souvent appelés, en particulier dans la littérature anglo-saxone, des modèles de bilan en eau, appeltation qui néglige le fait que ces modèles comprennent des équations dynamiques et pas seulement une équation de continuité (conservation de l'eau).

Les modèles existants ne sont pas très nombreux. Certains, assez sommaires, ont été mis au point pour une application particulière (SCHAAKE et CHUNZHEN, 1989), (McCABE et AYERS, 1989). D'autres résultent vraisemblablement d'une recherche heuristique assez longue sur leur architecture (ALLEY, 1985). Dans notre étude, nous avons porté notre recherche sur une adaptation au pas de temps mensuel du modèle GR3J (EDIJATNO et MICHEL, 1989), qui est un modèle global à trois paramètres fonctionnant au pas de temps journalier.

Nous nous sommes imposés de ne pas dépasser trois paramètres pour le modèle mensuel que nous appellerons, par analogie, GR3M. Cette contrainte est fondée sur les raisons suivantes:

- les données étant assez incertaines, on ne peut pas espérer s'en servir pour départager des versions sophistiquées :

- les données portant sur une durée trop courte pour constituer une série de contrồe différente de la série de calage, il faut éviter toute surparamétrisation ;

- si l'on veut espérer utiliser ultérieurement le modèle sur des bassins non jaugés, il faut au préalable établir des estimations a priori des paramètres, et l'on a jugé une telle opération condamnée à l'avance dans le cas d'une certaine redondance parmi les paramètres, redondance qui pourrait être perceptible à partir de trois paramètres, et devenir gênante au-delà. 


\section{6 - POINT DE DÉPART POUR L'ARCHITECTURE DU MODĖLE GR3M}

On peut se reporter à la publication précitée (EDIJATNO et MICHEL, 1989), pour une description sommaire du modèle GR3J qui a légèrement évolué depuis cette publication et dont nous éviterons une description complète pour alléger le présent article. Disons simplement qu'il repose sur trois opérateurs essentiels :

1] un réservoir * sol " qui conditionne la fonction de rendement selon une procédure exploitant le suivi de l' * état d'humidité « du système ;

2) un hydrogramme unitaire qui correspond à la partie « délai " de la fonction de transfert :

3) un réservoir de "routage "qui réalise la partie " tarissement " de la fonction de transfert. Chacun de ces opérateurs comporte le calage d'un seul paramètre.

Notons que les termes physiques employés ne correspondent pas à un désir de prise en compte de processus physiques mais seulement à une image conceptuelle qui permet d'affecter à chacun des composants du modèle un qualificatif facile à retenir.

Lorsque l'on passe du pas de temps journalier au pas de temps mensuel c'est la fonction de transfert qui est particulièrement affectée du fait que la partie * délai * disparaît. Avec elle, disparaît également un paramètre qui pourra être utilisé pour affiner la fonction de production. Sans rappeler les étapes intermédiaires, nous considérerons ici que nous sommes partis d'un modèle à 2 paramètres proche du modèle qui a servi de point de départ au modèle GR3J et qui ne comporte que les opérateurs 1) et 3). Son architecture est illustrée par la figure 2.

Ce modèle utilise comme seule entrée datée la pluie mensuelle sur le bassin considéré. Une autre donnée utilisée est l'évapotranspiration potentielle mensuelle calculée d'après une moyenne sur plusieurs années. Dans le cas qui nous intéresse, nous avons utilisé des données d'évaporation sur bac que nous avons réduites de $20 \%$ et qui proviennent de stations de mesure parfois assez éloignées du bassin versant considéré.

La fonction de production est assurée par une procédure de suivi de l'humidité du bassin versant. Cette a humidité " est représentée par le stock en eau S d'un réservoir « sol » qui est déprimé par l'évaporation potentielle d'une fraction de cette dernière, fonction de son taux de remplissage $s=S / A$, avec A premier paramètre du modèle. Ce même niveau de remplissage détermine également la fraction de la pluie destinée à l'écoulement et le réservoir « sol " absorbe le complément. La fraction de la pluie destinée à l'écoulement transite par un réservoir de « routage " dont la vidange est fonction de son contenu $\mathbf{R}$ et représente lécoulement mensuel :

$$
Q=\frac{R^{2}}{R+B}
$$

$B$ est le deuxième paramètre de ce premier modèle. 


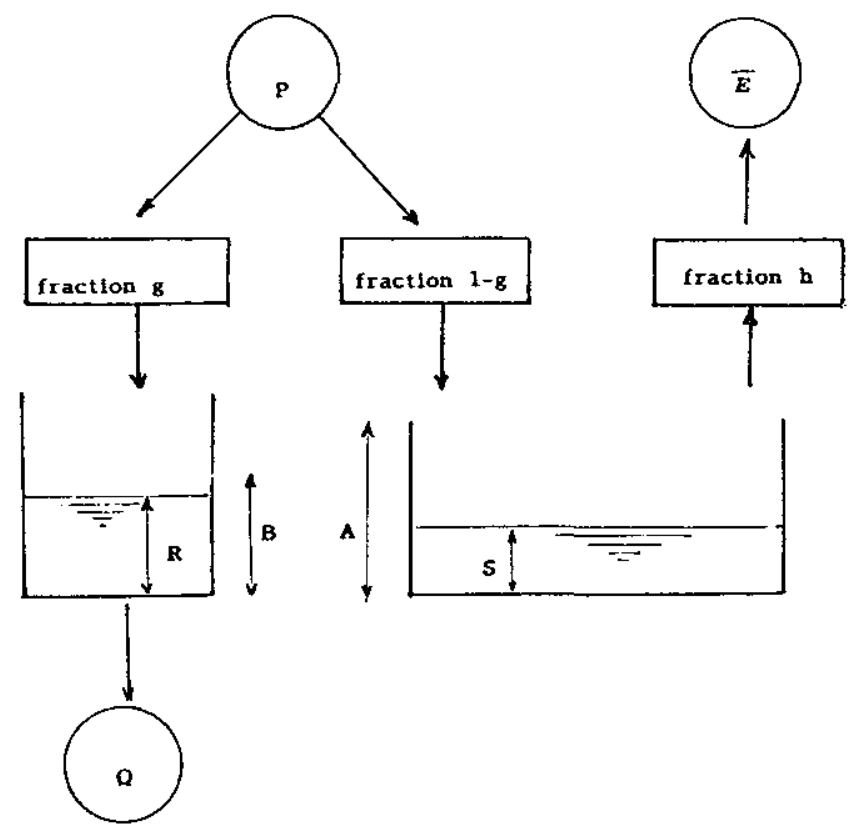

Flgure 2 Modèle à deux paramètres servant de point de départ pour le développement d'un modèle mensuel.

Two parameter model used for model development.
A, B : paramètre du modele
E : evapotranspiration potentielle mensuelle moyenne
g: $\mathbf{s}^{2}$
$\mathrm{h}: \mathrm{s}(2-\mathrm{s})$
$P$ : pluie mensuelle
$\mathrm{Q}:$ écoulement mensuel = $R^{2} /(R+B)$
$R$ : contenu du réservoir de * routage *
$S:$ contenu du réservoir * sol * s : S/A

\author{
A, $B$ : model parameters \\ $\bar{E}$ : averagod monthly \\ $g: s^{2}$ \\ evapotranspiration \\ $h: s(2-s)$ \\ $P$ : monthly rainfall \\ $Q$ : monthly runof $=$ \\ $A^{2} /(A+B)$ \\ $\boldsymbol{R}$ : routing resenvir storage \\ $S$ : soil reservoir storage \\ $s: S / A$
}

Cette architecture à deux réservoirs constitue le minimum nécessaire pour représenter simplement le comportement global d'un bassin versant. Les tentatives pour ne considérer qu'un seul réservoir conduisent en général à des résultats très sensiblement inférieurs ou nécessitent l'introduction de relations analytiques complexes comme dans le modèle a abcd „ (THOMAS, 1981), (ALLEY, 1985) que nous présenterons dans le paragraphe suivant. Comme cela a été dit précédemment, le schéma de la figure 2 ne résulte pas d'un choix a priori, reposant sur des concepts physiques. II a été retenu après de nombreuses confrontations avec des alternatives tout aussi simples mais qui se sont révélées moins efficaces pour simuler les sorties d'un bassin versant. 
Le présent modèle a été calé sur les 63 bassins versants évoqués précédemment en minimisant la variance de l'erreur mensuelle du modèle et a conduit à la valeur moyenne de $34,5 \%$ pour le critère déjà utilisé qui est le rapport de l'écart-type de l'erreur annuelle sur l'écoulement moyen interannuel. Ce dernier nombre est à rapprocher de la valeur obtenue par le modèle linéraire de $41,4 \%$. On constate un gain substantiel pour un nombre de paramètres identique.

\section{7 - PRÉSENTATION D'UN MOdĖle CONCURRENT, LE MODĖLE * ABCD * DE THOMAS (1981)}

THOMAS (1981) a proposé un modèle mensuel qu'il a appelé * abcd ", rappelant ainsi la présence de quatre paramètres. Nous en terons une présentation très proche de celle de GR3M pour faciliter la comparaison des deux modèles. La figure 3 permet d'illustrer les développements qui suivent. Un réservoir sol dont le contenu en eau est $S$ reçoit la pluie mensuelle $P$. II donne lieu à une pluie nette $P^{\prime}$ donnée par l'équation (4) :

$$
P^{\prime}=S\left[1-\frac{1}{1+s+\sqrt{\left(\frac{1+s}{2}\right)^{2}-B s}}\right]
$$

où :

$S=\frac{S}{A}=$ taux de remplissage du réservoir " sol "

$A$ et $B$ deux premiers paramètres du modèle avec $0 \leq B \leq 1$.

Lo niveau du réservoir sol devient donc $S^{\prime}$ :

$$
S^{\prime}=S+P-P^{\prime}
$$

Ensuite on prélève dans ce même réservoir l'évaporation réelle $\mathrm{E}^{\prime}$ :

$$
E^{\prime}=S^{\prime}[1-\exp (-E / A)]
$$

Une fraction $C$ de la pluie nette $P^{\prime}$ rentre dans un réservoir de routage linéaire (paramètre D) et le complément s'écoule directement à l'exutoire du bassin versant.

Ce modèle a èté comparé parmi d'autres au modèle GR3M. Mais aupravant nous allons présenter la suite des réflexions qui ont conduit au modèle GR3M définitif. 


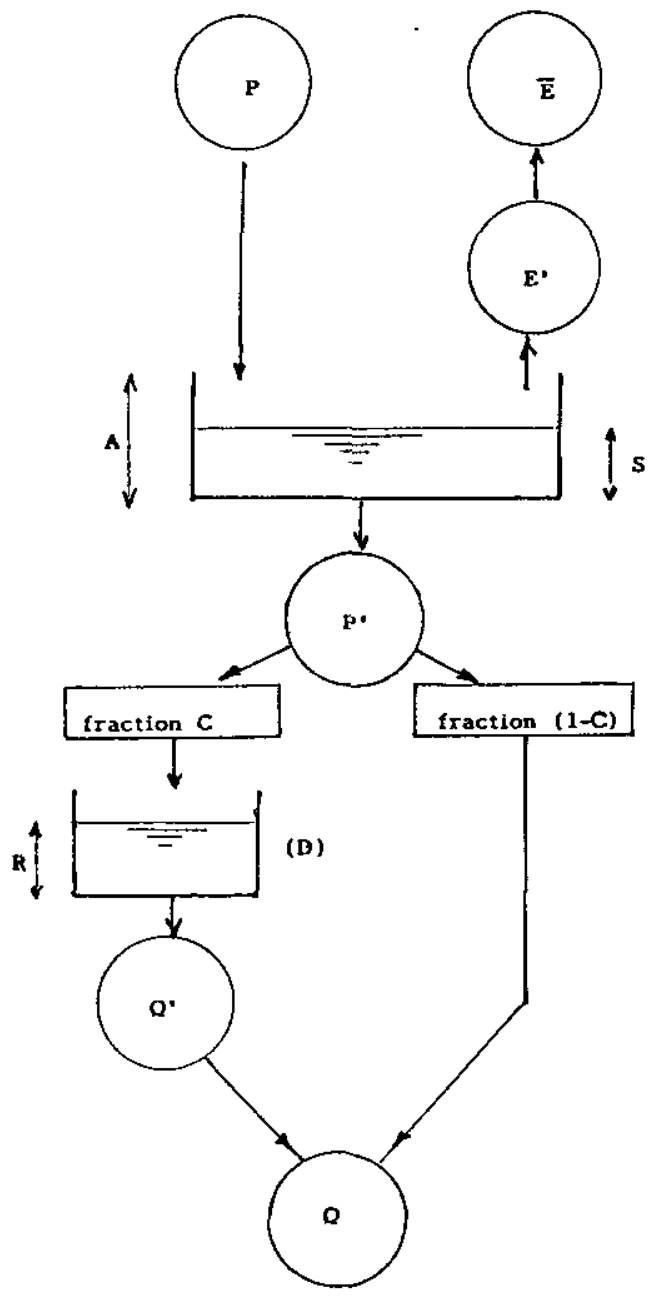

A, B : paramètre du modèle

$\vec{E}$ : évapotranspiration potentielle mensuelle moyenne

E' : évapotranspiration potentielis réelle

$P$ : pluie mensuelle

$P^{\prime \prime}$ : pluie nette mensuelle

$Q$ : écoulement mensuel

$Q^{*}$ : lacher du réservoir de * routage *

$R$ : stock en eau du réservoir de

$$
\text { "routage " }
$$

$S$ : stock en eau du réservoir * sol *

$S^{\prime}$ : stock intermédiaire du réservoir - sol .

$s$ : taux de remplissage du réservoir

$$
\text { * sol } *=S / A
$$

Figure 3 Le modèle "abcd" de THOMAS (1981)

* abcd " THOMAS' model (1981)
$A, B$ : model parameters
$\bar{E}$ : averaged monthly evapotranspiration
$E^{\prime}$ : actual evapotranspiration
$P$ : monthly rainfall
$P^{\prime}:$ monthly excess rainfall
$Q$ : monthly runoff
$Q^{\prime}$ : monthly release from the routing resenvir
$R$ : routing reservoir storage
$S$ : soil reservoir storage
$S^{\prime}$ : previous soil reservoir storage
$s$ : ratio of soil resenvir storage to soil reservoir capacity $=S / A$ 


\section{8 - RECHERCHE d'UN MOdĖle PLUS PRÉCIS}

On cherche dans ce paragraphe à améliorer le modèle en introduisant une spécification supplémentaire à laquelle on accepte de consacrer un paramètre au plus, noté $\mathrm{C}$ dans la suite de l'article.

Nous avons testé essentiellement six possibilités :

1) une adaptation de l'évaporation potentielle: $\bar{E} \rightarrow \bar{E}+C, C$ pouvant être positif ou négatif vient alors modifier l'évaporation potentielle ;

2) une réduction d'une entrée qui, si elie est positive, est une réduction de pluie et, si elle est négative, est une réduction d'évaporation :

$$
\text { si } C>0 \text { alors } P \rightarrow P-C \text {; si } C<0 \text { alors } \bar{E} \rightarrow \bar{E}-C
$$

3) une \& neutralisation » préalable portant sur une fraction $C$ de l'évaporation potentielle :

si $P>C$. $\bar{E}$ alors on considère une entrée $P-C E$ et une sortie potentielle $(1-$ C) $\bar{E}$

si $P<C$. $E$ alors on a seulement une sortie potentielle égale à $\bar{E}-$ $P$;

4) une modification du rendement de l'évaporation : la fraction $h$, rapport de l'évaporation réelle à l'évaporation potentielle, est liée au taux de remplissage du réservoir * sol * $(S=S / A)$ par :

$$
h=1-(1-s)^{c}
$$

5) une modification du rendement de la pluie : la fraction $\mathrm{g}$, rapport de la pluie nette à la pluie brute, est liée au taux de remplissage du réservoir " sol " $(S=S / A)$ par :

$$
\mathrm{g}=\mathrm{s}^{\mathrm{c}}
$$

6) un taux d'écoulement pseudo-direct (c'est-à-dire qui court-circuite le réservoir R) égal à $C$.

Les cinq premières transformations sont relatives à la fonction de rendement et la dernière est relative à la fonction de transfert.

Les six valeurs correspondantes du a critère annuel moyen » défini au paragraphe 5 sont respectivement :

$$
27,0,26,9,29,0,27,9,28,0 \text { et } 33,6
$$

Ainsi, l'utilisation la plus efficace de ce troisième paramètre consiste à introduire une diminution, soit de la pluie, soit de l'évaporation, d'une quantité fixe. Si nous cherchons à interpréter cette transformation, nous pouvons dire que dans le premier cas, on a une perte sur la pluie (infiltration profonde ?) et dans le deuxième cas, il n'y a pas d'évaporation sur une partie du bassin (absence d'humidité ?).

On a constaté sur un nombre non négligeable de bassins une valeur de $B$ nulle. Autrement dit, pour ces bassins il n'y a pas de report de l'écoulement d'un mois sur les suivants. Dans la version actuelle du modèle journalier, une fraction de $10 \%$ de la pluie destinée à l'écoulement court-circuite le réservoir R. Il semble naturel que cette fraction soit plus importante pour un pas de temps plus grand et pour un climat plus aride. Comme pour le modèle de bilan 
en eau de THORNTHWAITE (McCABE et AYERS, 1989), nous adopterons une fraction d'écoulement pseudo-direct égale à $50 \%$.

Le critère annuel moyen devient $26,5 \%$. La version correspondante consitue le modèle que nous retiendrons pour la simulation mensuelle, ce modèle est représenté sur la figure 4.

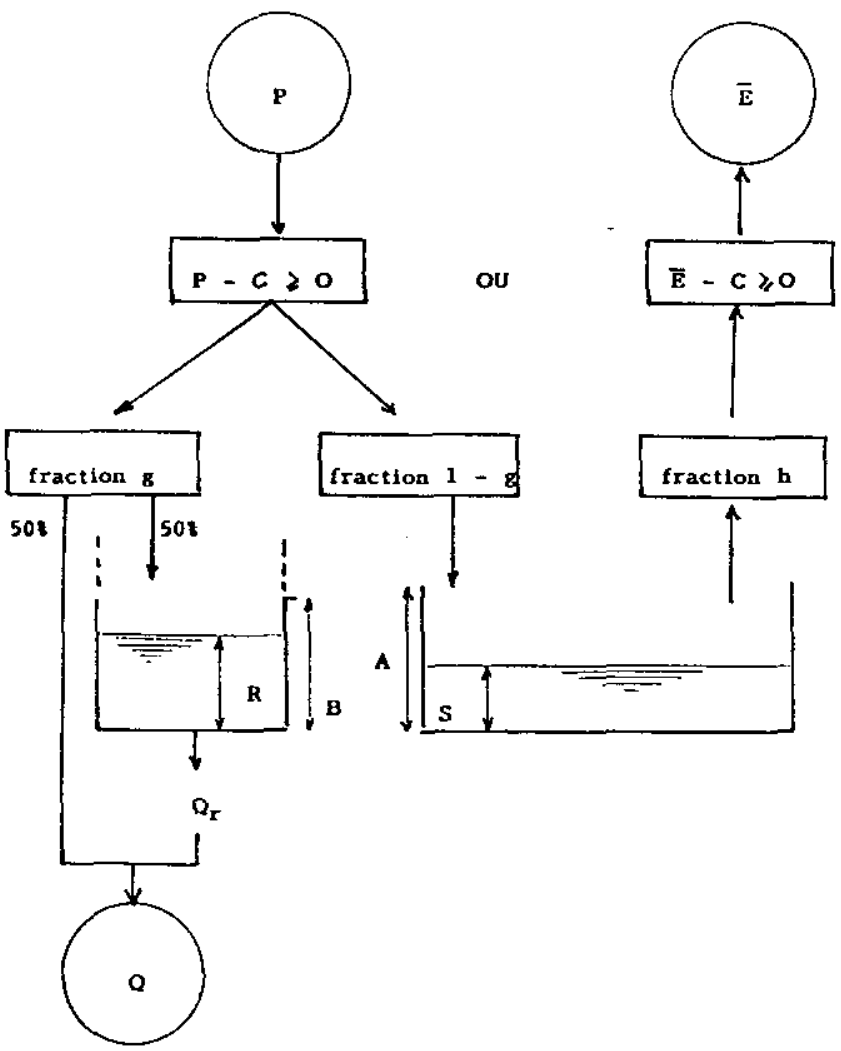

Figure 4 Schéma descriptif du modèle GR3M pour un mois donné.

Sketch of the GR3M model.

A, B, C : paramètres du modèle

$\bar{E}$ : évapotranspiration potentielle mensuelle moyenne

$\mathbf{g}, \mathbf{h}$ : fonctions du taux de remplissage du réservoir * sol * $g=s^{2}, h=s(2-s)$

$Q$ : écoulement mensuel

Qr: låcher du réservoir de * routage *

A : stock en eau du réservoir de " routage *

$S$ : stock en eau du réservoir * sol x

$s$ : taux de remplissage du réservoir " sol " (s = S/A)
A, $B, C$ : model parameters

$\bar{E}$ : averaged monthly evapotranspiration

$g, h$ : function of the soil reservoir storage rate $g=s^{2}, h=s(2-s)$

$Q:$ monthly runoff

Qr: routing reservoir release

$A$ : routing resenoir storage

$S$ : soil reservoir storage

$s$ : soil reservoir storage rate $(S=S / A)$ 
Pour éviter toute discontinuité dans le fonctionnement du modèle, la gestion du réservoir $S$ est effectuée en cinq étapes sur lesquelles sont également réparties les entrées. Le fonctionnement du réservoir $\mathrm{R}$ est réalisé en une seul étape : la pluie y est introduite puis la vidange est calculée avec la valeur de $\mathbf{R}$ ainsi modifiée. A titre de comparaison signalons que le modèle * abcd » (THOMAS, 1981) à quatre paramètres, aboutit à un critère égal à $27,0 \%$.

La progression du critère moyen entre le modèle linéaire, le modèle de base à deux paramètres et le modèle GR3M peut être complétée par la comparaison des distributions des erreurs standards obtenues par les trois modèles sur les 63 bassins versants, tel que montré à la figure 5 . On y montre également la distribution des erreurs standards du modèle * abcd ^ qui est très semblable à celle de GR3M. Précisons que c'est la moyenne de ces erreurs standards sur l'ensemble des 63 bassins versants qui constitue notre « critère annuel moyen ".

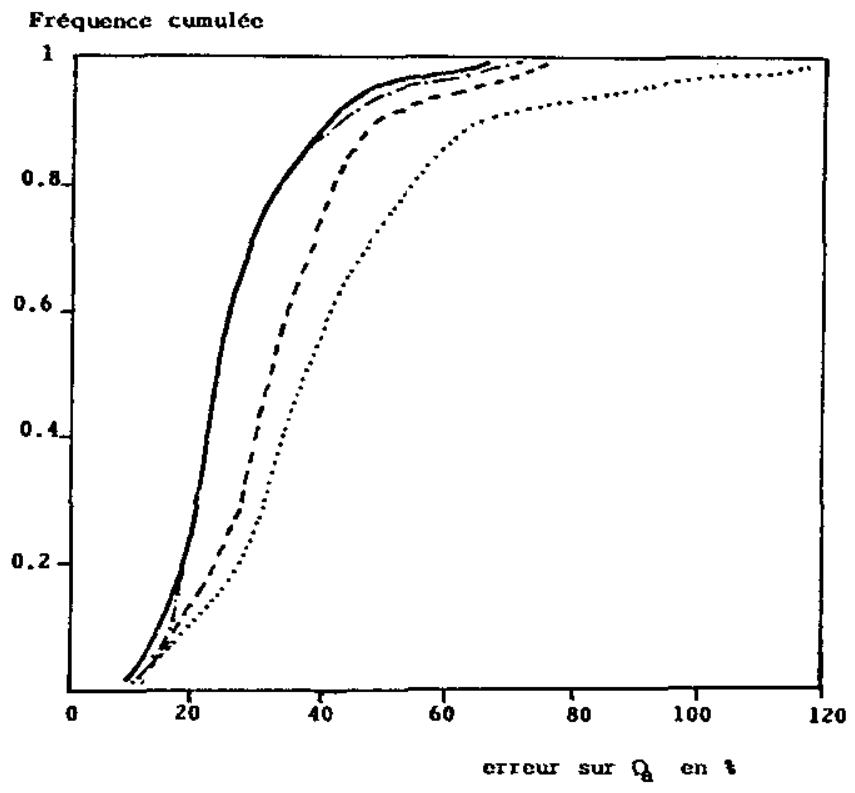

Figure 5 Comparaison des distributions des erreurs pour 4 modèles.

Comparison of the error distributions for 4 models.

...... modèle linéaire

- - - modèle à 2 paramètres

GR3M

— : - . mabcd (THOMAS)

axe des $x$ : erreur sur les Qa en $\%$ axe des y: Fréquence cumulée
...... linear model

_ _ _ two parameter model

- GR3M abcd (THOMAS)

$x$ - axis : annual flow error (\%)

$y$ - axis : cumulative frequency 
Le modèle GR3M supporte la comparaison avec différents autres modèles décrits dans la littérature hydrologique comme CREC (ROCHE, 1971), GARDENIA (THIERY, 1985), le modèle de BOUGHTON tel qu'il est présenté par MEIN et BROWN (1978) ou encore le modèle de THORNTHWATTE et MATHER (1955).

\section{9 - VALIDATION DU MODĖLE ET SENSIBILITÉ DES PARAMÈTRES}

Lès données disponibles étaient trop réduites pour réaliser une véritable validation sur un échantillon différent de celui du calage. On peut cependant avoir une idée de la consistance du modèle en effectuant une étude analytique de la sensibilité des paramètres (MEIN et BRONW, 1978), (KUCZERA, 1990). On peut présenter très simplement cette approche en se référant au modèle linéaire général.

Soit : $Q$ le vecteur des débits et soit $Q_{0}$ le vecteur des débits calculés par le modèle pour le jeu de paramètres optimal $(a, b, c)$ où les lettres minuscules repésentent les logarithmes des paramètres réels $A, B$ et $C$. Considérons une modification de $\varepsilon$ (nous prendrons $\varepsilon=0,05$ ) d'un des paramétres, a ou $b$ ou $c$. Soit $Q_{1}$ le vecteur des débits calculés avec le jeu $a+\varepsilon, b, c, Q_{2}$ le vecteur des débits calculés avec le jeu $(a, b+\varepsilon, c)$ et enfin : $Q_{3}$ le vecteur des débits calculés avec le jeu $(a, b, c+\varepsilon)$. Nous allons utiliser le modèle linéaire général pour relier l'erreur du modèle mensuel avec les variations de débits ainsi obtenues :

$$
Q-Q_{0}=\alpha \frac{Q_{1}-Q_{0}}{\varepsilon}+B \frac{Q_{2}-Q_{0}}{\varepsilon}+\gamma \frac{Q_{3}-Q_{0}}{\varepsilon}+u
$$

modèle où $\alpha, \beta, \gamma$ sont les variations optimales respectivement pour $a, b, c$, et $u$ est l'erreur de ce modèle linéaire.

L'intérêt de cette procédure n'est pas d'obtenir n'est pas d'obtenir les corrections $\alpha, \beta$ et $\gamma$ mais la matrice des variances-covariances de ces coefficients qui est approximativement celles des coefficients $\mathbf{a}, \mathbf{b}$ et $\mathbf{c}$. Cette démarche donne une idée de la précision des paramètres ainsi que des * liaisons * entre eux. Elle a été appliquée sur chacun des 63 bassins étudiés et révèle une assez bonne consistance pour les trois paramètres transformés a, b et c. Cela explique aussi la facilité avec laquelle la recherche dans l'espace $(a, b, c)$ aboutit sur le jeu optimal de paramètres. La méthode d'optimisation du type * descente maximale », développée pour le calage du modèle GR3J (EDIJATNO, MICHEL, 1990) ne présentant pas d'intérêt particulier, n'est pas exposée dans le cadre restreint de cet article. 


\section{0 - Quelques résultats Sur le modèle Gr3M}

Nous présentons dans le tableau 2 les résultats numériques pour le bassin du MAZAFRAN (code 021201) pour lequel les performances du modèle GR3M se situent dans la médiane, du moins pour le " critère annuel moyen ». C'est un bassin de $1900 \mathrm{~km}^{2}$ dont l'écoulement moyen interannuel est de $120 \mathrm{~mm}$. Les écarts-types des paramètres sont modérés et la matrice des corrélations des paramètres ne montre pas d'interdépendance majeure entre eux.

Tableau 2 Résultat du calage de GR3M sur le bassin du MAZAFraN.

Table 2 Result of calibration of the GR3M model upon the MAZAFRAN catchment.

\begin{tabular}{|c|c|c|c|c|c|c|c|c|c|c|c|c|}
\hline Superficie $\left(\mathrm{km}^{2}\right)$ & & & 1900 & & & & & & & & & \\
\hline Plie annuelle moyerne (mm) & & & 732 & & & & & & & & & \\
\hline Paramètre transformés a.b.c & & & 5.85 & & & 22 & -2 & .80 & & & & \\
\hline \multicolumn{13}{|l|}{ (C appliqué à la pluie) } \\
\hline \multicolumn{13}{|l|}{ Ecart-type des paramétres } \\
\hline \multirow[t]{2}{*}{$a b c$} & & & 0.15 & & & 68 & 0.2 & 24 & & & & \\
\hline & & & 1.00 & & & 0.79 & 0.6 & 62 & & & & \\
\hline \multirow[t]{2}{*}{ Matrice des corrélations } & & & -0.79 & & & .00 & -0 . & 10 & & & & \\
\hline & & & 0.62 & & & .10 & 1.0 & $\infty$ & & & & \\
\hline Erreur de bilan (\%) & & & $-4 \%$ & & & & & & & & & \\
\hline Critere annual moyen (\%) & & & 24 & & - & & & & & & & \\
\hline Nemerros des mois & 9 & 10 & 11 & 12 & 1 & 2 & 3 & 4 & 5 & 6 & 7 & 8 \\
\hline $\begin{array}{l}\text { Lames deeu mensuelles moyernes } \\
\text { observes (mm) }\end{array}$ & 1.5 & 2.5 & 9.2 & 14.4 & 24.8 & 30.5 & 33.1 & 20.4 & 8.2 & 21 & 1.0 & 0.5 \\
\hline $\begin{array}{l}\text { Lames d'eau mensubles moyernes } \\
\text { calcul'bes (mm) }\end{array}$ & 1.6 & 2.7 & 6.5 & 13.2 & 15.9 & 31.6 & 32.0 & 18.1 & 9.1 & 4.2 & 2.7 & 1.9 \\
\hline Erreurs standards $(\%)$ & 87 & 67 & 53 & 24 & 35 & 22 & 34 & 80 & 61 & 52 & $\pi$ & 89 \\
\hline
\end{tabular}

En ce qui concerne la variabilité des paramètres d'un bassin à l'autre, nous avons constaté comme intervalle interquartile pour $A(170,480) \mathrm{mm}$, pour $B$ $(15,360) \mathrm{mm}$ et pour $\mathrm{C}(1,7) \mathrm{mm}, \mathrm{C}$ étant un seuil soustractif appliqué aux pluies. Le seuil $\mathrm{C} n e$ s'applique à l'évaporation que dans un cas sur cinq environ (la transformée $\mathrm{C}$ est alors positive).

Sur les 63 bassins étudiés, $30 \%$ ont un coefficient d'écoulement annuel moyen inférieur à 0,1 . Ce sont des bassins très difficiles à modéliser et cela peut expliquer les valeurs parfois excessives du critère annuel moyen. 


\section{1 - DISCUSSION ET CONCLUSION}

L'objectif fixé initialement était de simuler les apports annuels d'un bassin versant, et pour atteindre cet objectif, nous avons constaté la nette supériorité des modèles conceptuels sur les modèles linéaires.

Nous pensons que ces modèles conceptuels ne doivent pas tirer leur validation de la présence d'équations d'inspiration physique, car leur intérêt est annulé par l'inévitable méconnaissance du système auquel elles sont appliquées, méconnaissance due au coût prohibitif d'un contrôle complet de tous les paramètres intervenant dans la description précise d'un bassin versant. Nous nous sommes astreints à construire, par une recherche systématique, un modèle mensuel rustique dépendant de trois paramètres.

Son application à un ensemble de 63 bassins en Algérie septentrionale a donné des résultats qui semblent aussi bons sinon meilleurs que ceux des modèles disponibles actuellement. Ce modèle élémentaire pourrait donner lieu à deux types d'applications :

- un essai d'explication des paramètres $A, B, C$, ou du moins leur distribution régionale, permettrait d'utiliser le modèle sur des bassins non jaugés :

- sur un bassin particulier, doté d'observations sur une longue durée, GR3M pourrait servir de base pour un modèle plus fin adapté à ce bassin. On pourra, entre autres, utiliser certaines des idées utilisées précédemment, pour améliorer le modèle de base à deux paramètres.

\section{REMERCIEMENTS}

Les auteurs remercient les relecteurs anonymes dont les critiques et les suggestions ont permis d'améliorer l'intérêt et la lisibilité du présent article.

\section{RÉFÉRENCES BIBLIOGRAPHIQUES}

ALLEY W.M., 1985. Water balance models in one-month-ahead streamflow forecasting. Water Resources Research, 21(4) : 597606.

BURGES S.J., LETTENMAIER, D.P., 1977. Comparison of annual streamflow models. Journal of Hydraulics Division, HY9 : 991 1006
EDIJATNO MICHEL, C., 1989. Un modèle pluie-débit joumalier à trois paramètres. $L a$ Houlle Blanche, 2 : 113-121.

GILROY E.J., HIRSH R.M., COHN T.A., 1990. Mean square error of regression-based constituent transport estimates. Water Resources Research, 26(9): 2069 2077. 
KUCZERA G., 1990. Assessing hydrologic model non linearity using response surface plots. Journal of hydrology, 118 : 143161.

MCCABE G.J., AYERS M.A., 1989. Hydrologic effects of climate change in the Delaware River Basin. Water Resources Bulletin. 25(6) : $1231-1242$.

MEIN R.G., BROWN B.M., 1978. Sensitivity of optimized parameters in watersheds models. Water Resources Research, 14(2): 299-303.

ROCHE M., 1971. Les divers types de modé les déterministes. La Houille Blanche (2) : 111-129.

SCHAAKE J.C. Jr., CHUNZHEN L., 1989. Development and application of simple water balance models to understand the rela- tionship between climate and water resources. LAHS, $181 ; 343-352$.

THIERY D., 1985. Analyse et modélisation des bassins versants de la Moselle française. BRGM, France.

THOMAS H.A., 1981. Improved methods for national water assessements. Report to U.S. water resources council. Contr. WR 15249270 . Washington.

THORNTHWAITE C., MATHER J., 1955. The water balance, publications in climatology, 8, C.W., Thornthwaite associates, Laboratory of climatology, Centerton, NJ.

TROUTMAN B.M., 1985. Errors and parameter estimation in precipitation runoff modeling. 1. theory. Water Resources Research, 21(3) : $1195-1213$. 